\title{
An ISO and IUE study of planetary nebula NGC 2440 *
}

\author{
J. Bernard Salas ${ }^{1,2}$, S. R. Pottasch ${ }^{2}$, W. A. Feibelman ${ }^{3}$, and P. R. Wesselius ${ }^{1}$ \\ 1 SRON Laboratory for Space Research, PO Box 800, NL 9700 AV Groningen, The Netherlands \\ 2 Kapteyn Astronomical Institute, PO Box 800, NL 9700 AV Groningen, The Netherlands \\ 3 Laboratory for Astronomy and Solar Physics, NASA-Goddard Space Flight Center, Greenbelt, MA 20771, USA
}

Received 13 November 2001 / Accepted 5 March 2002

\begin{abstract}
The infrared and ultraviolet spectra of planetary nebula NGC 2440 are presented. The observations were made by the Infrared Space Observatory (ISO) and the International Ultraviolet Explorer (IUE). These data, in conjunction with published optical observations have been used to derive electron temperature and density. The electron temperature increases with increasing ionization potential, from 11000 to $18000 \mathrm{~K}$. The electron density has a constant value of $4500 \mathrm{~cm}^{-3}$ in agreement with previous determinations. The chemical abundance has been derived for the following elements; helium, carbon, nitrogen, oxygen, neon, sulfur and argon. The ionization correction factor turns out to be very small for all species except sulfur.
\end{abstract}

Key words. ISM: abundances - planetary nebulae: individual: NGC 2440 - infrared: ISM - ISM: lines and bands

\section{Introduction}

NGC 2440 has a spectrum with lines of highly ionized elements. It is of great interest for different reasons: the nebula presents a complex bipolar or multi-polar morphology, effects of stratification (Walker \& Aller 1970), and strong N II lines (Shields et al. 1981). The latter leads to a high nitrogen abundance, that together with the high helium abundance, classify it as a type I nebula (Peimbert 1978). Studies by Shields et al. (1981) give an electron density of $3000 \mathrm{~cm}^{-3}$ and an electron temperature of 14000 using O III. Later studies by Hyung \& Aller (1998) lead to a higher density of $5000 \mathrm{~cm}^{-3}$ but agree on the electron temperature: $14200 \mathrm{~K}$ and $10000 \mathrm{~K}$ for O III and N II respectively.

ISO (Kessler et al. 1996) and IUE provide valuable information which allow us to derive more accurately the electron density $\left(N_{\mathrm{e}}\right)$, temperature $\left(T_{\mathrm{e}}\right)$ and nebular abundances. Infrared lines avoid many problems usually encountered when deriving abundances, which could be present in the nebula, especially temperature fluctuations. This and other advantages using infrared spectra are briefly discussed by Pottasch \& Beintema (1999).

The following section is devoted to the ISO and IUE observations. The nebula is larger than both the

Send offprint requests to: J. Bernard Salas,

e-mail: J.Bernard@sron.rug.nl

* Based on observations with ISO, an ESA project with instruments funded by ESA Member States (especially the PI countries: France, Germany, The Netherlands and the UK) and with the participation of ISAS and NASA.
ISO-SWS and IUE apertures and because of dust absorption (although small) the fluxes need to be corrected for extinction. This is discussed in Sect. 3. In Sect. 4 the line fluxes (infrared, optical and ultraviolet) are presented. The physical parameters, $N_{\mathrm{e}}$ and $T_{\mathrm{e}}$, for the nebula are derived and discussed in Sect. 5 .

In Sect. 6 a discussion of the chemical abundances is given. The conclusions are given in the last section.

\section{ISO and IUE observations}

The infrared observations were made with the Short Wavelength Spectrometer (SWS) on board ISO. Information about this instrument can be found in de Graauw et al. (1996). The wavelength range covered by the instrument is from 2.38 to $45.2 \mu \mathrm{m}$. The observation used corresponds to a SWS01 observing template, TDT number 72501762. This is a fast observation (Speed 2) of 1912 s exposure time, and was reduced at a fixed spectral resolution of 250 . The observations were centered at $\mathrm{RA}(2000) 07^{\mathrm{h}} 41^{\mathrm{m}} 55.4$ and $\operatorname{Dec}(2000)$ $-18^{\circ} 12^{\prime} 31.8^{\prime \prime}$. Pointing errors in ISO are uncertain by about $1.5^{\prime \prime}$. The position angle of this observation is $23^{\circ}$. In Fig. 1 the SWS spectrum of NGC 2440 is presented.

Standard reduction techniques were applied and no major problems were encountered. The reduction consists of; dark current subtraction, bad data removal (specially glitches which are important in band 4 from 29.0 to $45.2 \mu \mathrm{m})$; rebinning to a fixed resolution. It should be mentioned that the spectrum was not affected by fringes. Information about the tools used to reduce the data 


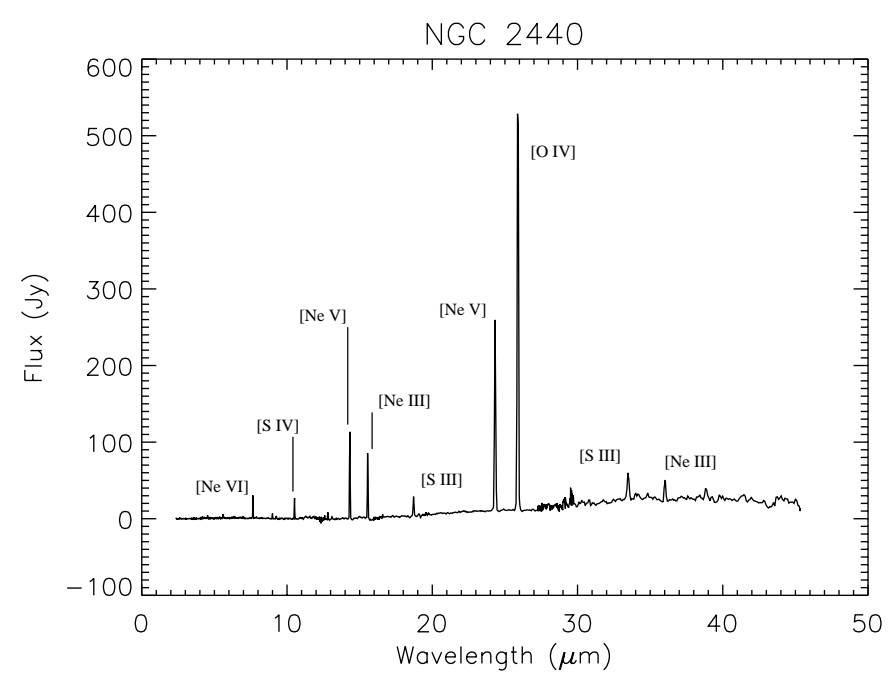

Fig. 1. SWS spectrum of NGC 2440. The continuum is very low. Strong lines are measured and have been labeled. Very weak PAH features are also present.

can be found in the interactive analysis software package distributed by the SWS consortium, IA3 (de Graauw et al. 1996). The observed infrared intensities are given in Table 2 .

Several ultraviolet observations exist, made with the International Ultraviolet Explorer (IUE). Two low resolution spectra and two high ones were used in this paper. The IUE observations were centered at the same position as the SWS observation within an accuracy of $2^{\prime \prime}$. The low resolution spectra are labeled SWP 07262 and LWR 06256 and the high ones as SWP 07263 and LWR 10741. The major axis position angle of the aperture for SWP07262 and SWP07263 was $72 \pm 3^{\circ}$. For LWP10741 it was $21 \pm 3^{\circ}$. The exposures were pointed at the 2000 coordinates $07^{\mathrm{h}} 41^{\mathrm{m}} 55.4,-18^{\circ} 12^{\prime} 32.7^{\prime \prime}$. The Ne V (157.5 nm) and Si II (171.1 and $180.8 \mathrm{~nm}$ ) lines were not present in these observations and were taken from Shields et al. (1981). The observed ultraviolet intensities are given in Table 3 .

\section{Corrections to line fluxes}

All fluxes measured: infrared, ultraviolet and optical, have been corrected for extinction (the correction in the infrared is very small). Because of the size of the nebula and relative small aperture sizes of the instruments, aperture corrections have been applied.

\subsection{Extinction}

In the previous work by Shields et al. (1981) a $C_{\mathrm{H}_{\beta}}=0.63$ was found (where $C_{\mathrm{H}_{\beta}}=\log \left(\frac{F\left(\mathrm{H}_{\beta}\right)_{\text {predicted }}}{\left.F\left(\mathrm{H}_{\beta}\right)_{\text {observed }}\right)}\right)$ ), although the value they determine by comparing the observed $I(\lambda 1640) / I(\lambda 4686)$ and $I(\lambda 2734) / I(\lambda 4686)$ ratio is 0.67 . This value is likely too high. In the recent paper by Hyung \& Aller (1998) a value of $C_{\mathrm{H}_{\beta}}=0.53$ is found by comparing the Balmer and Paschen line ratios.
In this work the observed lines have been corrected for extinction using $C_{\mathrm{H}_{\beta}}=0.50$. This leads to $E_{B-V}=0.34$.

To measure the extinction the predicted $F_{\mathrm{H}_{\beta}}$ given by radio fluxes has been determined. Applying the following equation:

$$
F\left(\mathrm{H}_{\beta}\right)=\frac{S_{\nu}}{2.82 \times 10^{9} t^{0.53}\left(1+\frac{\mathrm{He}^{+}}{\mathrm{H}^{+}}+3.7 \frac{\mathrm{He}^{++}}{\mathrm{H}^{+}}\right)}
$$

the expected $\mathrm{H}_{\beta}$ flux can be found. In Eq. (1) $S_{\nu}=$ $0.411 \mathrm{Jy}$ at $5 \mathrm{GHz}$ (Pottasch 1984), $t$ is the temperature of the nebula in $10^{4} \mathrm{~K}$ (in this case $T_{\mathrm{e}}=15000$ is used) and $2.82 \times 10^{9}$ is a conversion factor if the flux density $S_{\nu}$ is in Jy and the $F\left(\mathrm{H}_{\beta}\right)$ in erg $\mathrm{cm}^{-2} \mathrm{~s}^{-1}$. The helium abundances are taken from Table 7 and have been derived using the theoretical predictions of Benjamin et al. (1999) at a $N_{\mathrm{e}}=10000 \mathrm{~cm}^{-3}$ (using $N_{\mathrm{e}}=100 \mathrm{~cm}^{-3}$ will lead to the same values). Equation (1) leads to a predicted $\mathrm{H}_{\beta}$ of $91.0 \times 10^{-12} \mathrm{erg} \mathrm{cm}^{-2} \mathrm{~s}^{-1}$. The extinction factor is thus $C_{\mathrm{H}_{\beta}}=0.46$. This is in good agreement with what Hyung \& Aller (1998) $\left(C_{\mathrm{H}_{\beta}}=0.53\right)$ found. An average of those two $\left(\overline{C_{\mathrm{H}_{\beta}}}=0.50\right)$ has been used in the present paper. The actual correction was applied using the Fluks et al. (1994) law.

\subsection{Aperture corrections}

NGC 2440 has a measured size (Shields et al. 1981) of 10" for the main body and a faint envelope that extends up to $30^{\prime \prime}$. ISO-SWS uses different size apertures for the different bands. These sizes range from $14^{\prime \prime} \times 20^{\prime \prime}$ (the smallest, measuring the 2.38 to $12.0 \mu \mathrm{m}$ wavelength interval), to larger apertures $14^{\prime \prime} \times 27^{\prime \prime}(12.0-27.5 \mu \mathrm{m}), 20^{\prime \prime} \times 27^{\prime \prime}(27.5$ $29.0 \mu \mathrm{m})$ and $20^{\prime \prime} \times 33^{\prime \prime}(29.0-45.2 \mu \mathrm{m})$. The aperture of the IUE is very similar to ISO-SWS smallest aperture, it's an ellipse of $10^{\prime \prime} \times 23^{\prime \prime}$. It is likely that some of the nebula is missing and, because of the similarity in aperture size, the correction factors in the infrared and ultraviolet will be similar. Due to the fact that the nebula is larger than the smallest ISO apertures one should expect jumps in the SWS spectrum caused by the different aperture sizes used. This is not the case, as can be seen in Fig. 1, because the contribution of the continuum at lower wavelengths (smallest apertures) in this nebula is practically zero. There is a real (see Fig. 1) contribution of the nebula to the continuum at longer wavelengths, starting at around $21 \mu \mathrm{m}$. Since no jumps are seen in that part of the spectrum, the total nebular flux has probably been measured above $12 \mu \mathrm{m}$.

In order to correct the infrared lines for aperture effects the SWS measurements have been compared with those from the Infrared Astronomical Satellite (IRAS), which had an aperture big enough to contain the whole nebula. This comparison is shown in Table 1. The SWS and IRAS fluxes (Pottasch et al. 2000) are shown in Cols. 4 and 5. In the last column the scale factor to reproduce the IRAS measurements (basically $F^{\text {iras }} / F^{\text {sws }}$ ) is given. 
Table 1. Comparison of IRAS and ISO-SWS fluxes, in units of $10^{-12} \mathrm{erg} \mathrm{cm}^{-2} \mathrm{~s}^{-1}$.

\begin{tabular}{clcccc}
\hline \hline $\begin{array}{c}\lambda \\
(\mu \mathrm{m})\end{array}$ & Ion & \multicolumn{2}{c}{ SWS } & IRAS & IRAS/SWS \\
\cline { 3 - 4 } & & Aperture & Flux & & \\
& & & \\
& & & \\
7.654 & Ne VI & $14^{\prime \prime} \times 20^{\prime \prime}$ & 52 & $70:$ & 1.35 \\
10.52 & S IV & $14^{\prime \prime} \times 20^{\prime \prime}$ & 29 & 50 & 1.72 \\
12.81 & NeII & $14^{\prime \prime} \times 27^{\prime \prime}$ & 9.2 & 8 & 0.87 \\
14.32 & Ne V & $14^{\prime \prime} \times 27^{\prime \prime}$ & 96 & 90 & 0.93 \\
15.56 & Ne III & $14^{\prime \prime} \times 27^{\prime \prime}$ & 65 & 75 & 1.15 \\
18.70 & S III & $14^{\prime \prime} \times 27^{\prime \prime}$ & 17 & $30:$ & 1.80 \\
\hline
\end{tabular}

The advantage of this is that the lines IRAS has measured have been measured by the SWS using different apertures (see above) so that a correction for each one can be applied. As can be seen in Table 1 the IRAS fluxes for NeVI and SIV are bigger than the fluxes measured with SWS. This means that some flux is missing in the smaller apertures. That is not the case for the ions longward of $12 \mu \mathrm{m}$ which have been measured with a larger aperture. The difference in the fluxes is within the errors expected. Only the S III seems not to agree but the IRAS flux reported is uncertain. From the Ne II, Ne III and $\mathrm{NeV}$ lines it appears that no flux is missing in these apertures. Therefore, all measurements with the large SWS aperture seem to see almost all the nebular line flux emission. In the smallest apertures some flux is missing. The aperture correction factor are 1.35 and 1.72 for Ne VI and $\mathrm{S}$ IV. Although the Ne VI line from IRAS is noisy, combining these two results lead to an average correction factor of 1.54 .

Another way to estimate the "missing aperture" factor is by predicting the $\mathrm{H}_{\beta}$ flux from the $\mathrm{Br}_{\alpha}$ line. The dereddened $\mathrm{Br}_{\alpha}$ flux is $4.00 \times 10^{-12} \mathrm{erg} \mathrm{cm}^{-2} \mathrm{~s}^{-1}$. The theoretical prediction given by Hummer \& Storey (1987) at $T_{\mathrm{e}}=15000 \mathrm{~K}$ and $N_{\mathrm{e}}=1000 \mathrm{~cm}^{-3}$ (using $N_{\mathrm{e}}=10000 \mathrm{~cm}^{-3}$ will lead to the same conclusions) leads to $I\left(\mathrm{Br}_{\alpha} / \mathrm{H}_{\beta}\right)=6.86$. A flux of $\mathrm{H}_{\beta}$ of $58.3 \times$ $10^{-12} \mathrm{erg} \mathrm{cm}^{-2} \mathrm{~s}^{-1}$ will follow from it. However, the predicted $\mathrm{H}_{\beta}$ flux is $91.0 \times 10^{-12} \mathrm{erg} \mathrm{cm}^{-2} \mathrm{~s}^{-1}$ ). This leads to an aperture correction factor of 1.56 is which agrees with the average factor derived before. Therefore a factor of 1.56 has been adopted to correct the measurements done in the smallest SWS aperture (below $12 \mu \mathrm{m}$ ).

To correct the ultraviolet lines the theoretical prediction (Hummer \& Storey 1987) of the ratio of the helium lines $F(\lambda 1640) / F(\lambda 4686)=6.94$ (for a $T_{\mathrm{e}}=15000 \mathrm{~K}$ ) has been used. Using the dereddened fluxes listed in Tables 3 and 4 a ratio of 3.54 is found. The scaling factor becomes 1.96 , which we shall use.

The optical data used in this study has been taken from Hyung \& Aller (1998). These fluxes only represent part of the nebula, specifically the brightest part of the northern blob using a slit of $4^{\prime \prime}$. Using the entire $\mathrm{H}_{\beta}$ flux, they have been scaled to the total flux.
Table 2. Infrared line intensities for NGC 2440, fluxes in units of $10^{-12} \mathrm{erg} \mathrm{cm}^{-2} \mathrm{~s}^{-1}$.

\begin{tabular}{|c|c|c|c|c|}
\hline \multirow{2}{*}{$\begin{array}{c}\lambda \\
(\mu \mathrm{m})\end{array}$} & \multirow[t]{2}{*}{ Ident. } & \multicolumn{3}{|c|}{ Flux } \\
\hline & & observed & dereddened $^{1}$ & corrected $^{2}$ \\
\hline 4.051 & H г $\mathrm{Br} \alpha$ & 3.91 & 4.00 & 6.24 \\
\hline 4.527 & [Ar VI] & 16.8 & 17.2 & 26.8 \\
\hline 5.611 & {$[\mathrm{Mg} \mathrm{V}]$} & 13.5 & 13.7 & 16.09 \\
\hline 6.986 & {$[$ Ar II $]$} & 3.30 & 3.34 & 5.21 \\
\hline 7.654 & {$[\mathrm{Ne}$ VI] } & 52.0 & 52.7 & 82.2 \\
\hline 7.900 & {$[\mathrm{Ar} \mathrm{V}]$} & 4.33 & 4.40 & 6.86 \\
\hline 8.994 & [Ar III] & 9.37 & 9.83 & 15.33 \\
\hline 10.512 & {$[\mathrm{~S}$ IV $]$} & 29.1 & 30.5 & 57.6 \\
\hline 12.813 & {$[\mathrm{Ne}$ II $]$} & 9.18 & 9.36 & 9.36 \\
\hline 13.101 & {$[\mathrm{Ar} \mathrm{V}]$} & 4.20 & 4.28 & 4.28 \\
\hline 14.323 & {$[\mathrm{Ne} \mathrm{V}]$} & 96.4 & 97.8 & 97.8 \\
\hline 15.557 & [Ne III $]$ & 65.2 & 66.3 & 66.3 \\
\hline 18.712 & [S III] & 16.7: & 17.1 & 17.1 \\
\hline 24.321 & {$[\mathrm{Ne} \mathrm{V}]$} & 121.8 & 123.5 & 123.5 \\
\hline 25.896 & {$[\mathrm{O}$ IV $]$} & 297.9 & 301.7 & 301.7 \\
\hline 33.479 & [S III] & 14.8: & 14.9 & 14.9 \\
\hline 36.017 & [Ne III $]$ & 9.35 & 9.42 & 9.42 \\
\hline
\end{tabular}

: Noisy line.

${ }^{1}$ Dereddened fluxes using $C_{\mathrm{H}_{\beta}}=0.50$.

2 Aperture corrected and dereddened fluxes: only for lines below $12 \mu \mathrm{m}$ an aperture correction factor of 1.56 has been applied.

\section{Line fluxes}

In Tables 2-4 the infrared, ultraviolet and optical lines are listed.

\subsection{Infrared spectrum}

The infrared spectrum as measured by ISO-SWS is shown in Fig. 1. The spectrum is especially rich in neon and sulfur lines which are also the strongest, after the O IV line at $25.9 \mu \mathrm{m}$. Seventeen lines have been measured (Table 2). This is shown in Table 2. Only one hydrogen line $\left(\mathrm{Br}_{\alpha}\right)$ and one helium line at $9.26 \mu \mathrm{m}$ are found. High ionization stages for argon (Ar VI), neon (Ne VI) and magnesium $(\mathrm{Mg} \mathrm{V})$ have been measured. The strongest line is that of O IV. Argon, neon and sulfur are all present in the most important stages of ionization.

Calibration errors increase at longer wavelength, but on average they amount to about $20 \%$. Random errors depend on the line strength, and they decrease with line strength. Normally weak lines $(I<5$, where $I$ is in $10^{-12} \mathrm{erg} \mathrm{cm}^{-2} \mathrm{~s}^{-1}$ ) have estimated errors of about $20 \%$, intermediate lines $(5<I<10)$ of about $10 \%$, while the strongest lines $(I>10)$, have normally less than $5 \%$ uncertainty. There are just two exceptions, the sulfur lines at 33.479 and $18.712 \mu \mathrm{m}$. In the latter, the up and down scans differ by $40 \%$. 
Table 3. IUE intensities, fluxes in units of $10^{-12} \mathrm{erg}_{\mathrm{cm}}^{-2} \mathrm{~s}^{-1}$. The dereddened and aperture corrected fluxes in Cols. 5 and 6 correspond to the low resolution spectra.

\begin{tabular}{|c|c|c|c|c|c|c|c|c|c|c|c|}
\hline \multirow{2}{*}{$\begin{array}{c}\lambda \\
(\mathrm{nm})\end{array}$} & \multirow[t]{2}{*}{ Ident. } & \multicolumn{2}{|c|}{ Flux $^{1}$} & \multirow[t]{2}{*}{ Flux $^{2}$} & \multirow[t]{2}{*}{ Flux $^{3}$} & \multirow{2}{*}{$\begin{array}{c}\lambda \\
(\mathrm{nm})\end{array}$} & \multirow[t]{2}{*}{ Ident. } & \multicolumn{2}{|c|}{ Flux $^{1}$} & \multirow[t]{2}{*}{ Flux $^{2}$} & \multirow[t]{2}{*}{ Flux ${ }^{3}$} \\
\hline & & $\mathrm{HR}$ & LR & & & & & HR & LR & & \\
\hline 116.86 & $\mathrm{C}_{\text {IV }}$ & 3.60 & & 99.92 & 195.85 & 180.80 & Si II & 0.160 & & 1.82 & $3.56^{*}$ \\
\hline 123.92 & $\mathrm{~N} \mathrm{v}$ & 1.33 & 2.74 & 57.99 & 113.66 & 190.70 & C III] & 24.3 & 41.9 & 517.7 & 1014 \\
\hline 124.31 & $\mathrm{~N} \mathrm{v}$ & 1.45 & & & & 190.91 & C III] & 17.7 & & & \\
\hline 130.06 & C III & & 0.39 & 6.95 & 13.62 & 229.80 & C III & 0.26 & 0.61 & 9.58 & 18.78 \\
\hline 132.77 & C II & & 0.42 : & 6.95 & 13.62 & 232.65 & C II] & 1.46 & 3.7 & 21.8 & 42.8 \\
\hline 137.09 & $\mathrm{Ov}$ & 0.55 & 0.32 & 4.79 & 9.39 & 232.81 & C II] & 0.65 & & 9.67 & 18.95 \\
\hline 140.13 & O IV] & 0.78 & 2.74 & 38.5 & 75.5 & 238.66 & He II & 0.31 & & 3.90 & 7.63 \\
\hline 140.51 & O IV] & 0.57 & & & & 242.30 & {$[\mathrm{Ne}$ IV $]$} & 3.38 & 7.47 & 85.3 & 167.1 \\
\hline 140.73 & O IV] & 0.28 & & & & 242.56 & {$[\mathrm{Ne} I \mathrm{I}]$} & 3.79 & & & \\
\hline 148.36 & N IV] & 3.75 & 10.14 & 130.6 & 256.0 & 247.15 & {$[\mathrm{O}$ II] } & 0.56 & 1.04 & 10.76 & 21.1 \\
\hline 148.68 & $\mathrm{~N} \mathrm{IV]}$ & 2.38 & & & & 251.24 & He II & 0.58 & 1.04 & 9.99 & 19.59 \\
\hline 154.86 & C IV & 11.99 & 27.4 & 349.1 & 684.2 & 273.46 & He II & 1.13 & 1.62 & 11.34 & 22.2 \\
\hline 155.12 & C IV & 7.24 & & & & 278.42 & {$[\mathrm{Mg} \mathrm{v}]$} & 0.66: & 0.87 & 5.86 & 11.48 \\
\hline 157.50 & $\mathrm{Ne} \mathrm{V}$ & 0.630 & & 8.05 & $15.78^{*}$ & 279.73 & Mg II & 0.23 & & 1.53 & 3.01 \\
\hline 160.24 & {$[\mathrm{Ne}$ IV] } & & 0.58 & 7.37 & 14.44 & 280.45 & Mg II & 0.13 & & 0.86 & 1.69 \\
\hline 164.07 & Не II & 22.4 & 23.4 & 289.7 & 567.9 & 283.00 & O IV? & 0.20 & & 1.30 & 2.55 \\
\hline 166.10 & O III] & 0.44 & 2.96: & 35.6 & 69.7 & 283.77 & O III & 0.72 & 0.59 & 3.82 & 7.48 \\
\hline 166.64 & O III] & 1.06 & & & & 285.51 & $\mathrm{Mg}_{\mathrm{I}}$ & 0.31 & & 1.98 & 3.88 \\
\hline 171.10 & Si II & 0.630 & & 7.36 & $14.43^{*}$ & 286.96 & $?$ & 0.13 & & 0.82 & 1.61 \\
\hline 171.68 & S III? & & 0.42 & 4.89 & 9.59 & 290.14 & C IV & 0.12 & & 0.74 & 1.46 \\
\hline 174.71 & N III] & 0.48 & 10.1 & 115.6 & 226.6 & 292.95 & {$[\mathrm{Mg} \mathrm{v}]$} & 0.21 & & 1.27 & 2.50 \\
\hline 174.90 & N III] & 0.71 & & & & 302.50 & O III & 0.21 & 0.15 & 0.86 & 1.68 \\
\hline 175.00 & N III] & 4.07 & & & & 304.86 & O III & 1.03 & 1.22 & 6.88 & 13.49 \\
\hline 175.24 & N III] & 2.19 & & & & 313.44 & O III & 7.15 & 7.88 & 42.4 & 83.0 \\
\hline 175.43 & N III] & 0.45 & & & & 320.47 & Не II & 2.69 & 3.88 & 20.10 & 39.4 \\
\hline 180.49 & [Ne III] & & 0.45 & 5.11 & 10.01 & & & & & & \\
\hline
\end{tabular}

Flux $^{1}$ : Observed flux; HR $=$ High Resolution Spectra; LR $=$ Low Resolution Spectra.

Flux ${ }^{2}$ : Dereddened flux.

Flux ${ }^{3}$ : Dereddened and aperture corrected flux (Aperture factor is 1.96).

* Taken from Shields et al. (1981).

: Noisy.

\subsection{Ultraviolet spectrum}

In the IUE spectra fifty lines were measured. Nitrogen, oxygen, carbon and magnesium lines are abundant (Table 3). The low resolution spectrum was used when deriving the physical parameters and abundance; if not available, then the high resolution spectrum was used, and if not possible data from Shields et al. (1981) was used. The sum of the high resolution doublet fluxes should be the same as the unresolved low resolution, but often is not. Low-dispersion SWP spectra are preferred because they have the highest accuracy. In the high-dispersion observations the exposures are generally much longer and thus subject to higher background radiation noise. In addition, echelle order splice points and less well defined continuum levels lead to greater uncertainties in flux determination. A nominal error of $\pm 10 \%$ was assumed for well exposed (unsaturated) IUE low-dispersion spectra ( 200 DN).

\subsection{Optical data}

Together with the infrared and ultraviolet data, optical lines were also used to derive the physical parameters of the nebula, and to have information on some unobserved stages of ionization. The observed fluxes were taken from Hyung \& Aller (1998), and corrected for reddening. The Ne v line flux given by Rowland et al. (1993) was also used. Table 4 lists the optical lines used.

For N II line fluxes at 575.5 and $658.4 \mathrm{~nm}$, given by Perinotto \& Corradi (1998), have been included. They measure the flux in different regions of the nebula: from the center $(c e n)$, to positive increasing distances from the center $(p 1, p 2$, etc.) and negative increasing distances ( $n 1$, n2, etc.), relative to the orientation defined by the position angle of their observations (see Perinotto \& Corradi 1998 for details). The average values used in this paper correspond to the $c e n+p 1+n 1$ regions which agree with the region observed by ISO-SWS and IUE. 
Table 4. Optical line fluxes. Observed values were taken from Hyung \& Aller (1998). The dereddened fluxes were derived using an interstellar extinction of $C_{\mathrm{H}_{\beta}}=0.50\left(E_{B-V}=0.34\right)$. Fluxes are in units of $10^{-12} \mathrm{erg} \mathrm{cm}^{-2} \mathrm{~s}^{-1}$.

\begin{tabular}{|c|c|c|c|}
\hline $\begin{array}{l}\text { Wavelength } \\
\quad(\mathrm{nm})\end{array}$ & Ident. & $\begin{array}{l}\text { Measured } \\
\text { Fluxes }\end{array}$ & $\begin{array}{l}\text { Dereddened } \\
\text { Fluxes }\end{array}$ \\
\hline 342.68 & {$[\mathrm{Ne} \mathrm{V}]$} & $25.3^{*}$ & 118 \\
\hline 372.60 & {$[\mathrm{O}$ II $]$} & 15.17 & 64.1 \\
\hline 372.97 & {$[\mathrm{O}$ iI $]$} & 7.59 & 32.0 \\
\hline 386.87 & [Ne III] & 27.2 & 111.3 \\
\hline 436.32 & [O III] & 7.29 & 26.6 \\
\hline 447.15 & $\mathrm{He}$ I & 0.99 & 3.51 \\
\hline 468.57 & He II & 23.5 & 78.1 \\
\hline 471.15 & [Ar IV] & 2.29 & 7.56 \\
\hline 474.00 & [Ar IV] & 2.46 & 8.05 \\
\hline 486.10 & $\mathrm{H}_{\beta}$ & 31.6 & 99.54 \\
\hline 500.68 & [O III] & 522.4 & 1572 \\
\hline 519.17 & [Ar III] & 0.10 & 0.28 \\
\hline 519.80 & {$\left[\mathrm{~N}_{\mathrm{I}}\right]$} & 1.69 & 4.81 \\
\hline 520.03 & {$[\mathrm{~N} \mathrm{I}]$} & 1.17 & 3.33 \\
\hline 551.76 & [Cl III] & 0.64 & 0.52 \\
\hline 553.79 & [Cl III] & 0.23 & 0.60 \\
\hline 575.46 & {$[\mathrm{~N}$ II $]$} & 4.80 & 12.02 \\
\hline 575.46 & {$[\mathrm{~N}$ II $]$} & $4.54^{\dagger}$ & 11.37 \\
\hline 587.57 & He I & 3.10 & 7.60 \\
\hline 630.03 & {$[\mathrm{O} \mathrm{I}]$} & 6.53 & 14.9 \\
\hline 631.20 & [S III] & 0.66 & 1.50 \\
\hline 658.33 & {$[\mathrm{~N}$ II $]$} & 383.7 & 841 \\
\hline 658.33 & {$[\mathrm{~N}$ II $]$} & $263.1^{\dagger}$ & 576.7 \\
\hline 671.64 & {$[\mathrm{~S}$ II] } & 1.96 & 4.21 \\
\hline 673.08 & {$[\mathrm{~S}$ II $]$} & 3.13 & 6.71 \\
\hline 700.59 & {$[\mathrm{Ar} \mathrm{V}]$} & 1.39 & 2.87 \\
\hline 726.29 & [Ar IV] & 0.149 & 0.296 \\
\hline 732.00 & [O II] & 3.14 & 6.196 \\
\hline 733.03 & {$[\mathrm{O} \quad \mathrm{II}]$} & 2.63 & 5.18 \\
\hline
\end{tabular}

* Taken from Rowland et al. (1993).

$\dagger$ Taken from Perinotto \& Corradi (1998) (see text).

\section{Physical parameters}

In this section the electron temperature and density are analyzed.

\subsection{Analysis}

\subsubsection{Electron density}

$N_{\mathrm{e}}$ has been derived for nine ions. A $T_{\mathrm{e}}=15000 \mathrm{~K}$ has been assumed and a justification for this choice is given in the following subsection. The $N_{\mathrm{e}}$ is shown in Table 5 , and as can be seen, $N_{\mathrm{e}}$ seems to be constant for all the ions except for S III and ArV. For comparison, the $N_{\mathrm{e}}$ found by Keenan et al. using the $R$-matrix method in successive papers in 1996, 1997, 1998 and 1999 for S II, Ar IV, Ne IV and O II respectively have been included in the table, which average to $4000 \mathrm{~cm}^{-3}$. Hyung \& Aller (1998) give an average $N_{\mathrm{e}}$ of $5000 \mathrm{~cm}^{-3}$. If the average $N_{\mathrm{e}}$ is derived from Table 5, excluding SIII and ArV, a value of $4500 \mathrm{~cm}^{-3}$ is found. This is in good agreement with
Table 5. Electron density. $T_{\mathrm{e}}=15000 \mathrm{~K}$ is used.

\begin{tabular}{lccccc}
\hline \hline Ion & $\begin{array}{c}\text { IP }^{\star} \\
(\mathrm{eV})\end{array}$ & $\begin{array}{c}\text { Lines used } \\
(\mathrm{nm})\end{array}$ & $\begin{array}{c}\text { Obs. } \\
\text { ratio }\end{array}$ & $\begin{array}{c}N_{\mathrm{e}} \\
\left(\mathrm{cm}^{-3}\right)\end{array}$ & $\begin{array}{c}\text { Other } \\
\text { ref. }^{1}\end{array}$ \\
\hline S II & 10.4 & $673.1 / 671.6$ & 1.59 & 4260 & $3100^{a}$ \\
O II & 13.6 & $372.6 / 372.9$ & 2.00 & 4890 & $5000^{d}$ \\
S III & 23.3 & $33470 / 18700$ & 0.87 & $871:$ & \\
Cl III & 23.8 & $553.8 / 551.8$ & 1.15 & $4900:$ & \\
C III & 24.4 & $190.7 / 190.9$ & 1.35 & 4360 & \\
Ar IV & 40.7 & $472.4 / 472.5$ & 1.04 & 4900 & \\
Ar IV & 40.7 & $471.1 / 474.0$ & 0.94 & 5200 & $4000^{b}$ \\
Ar V & 59.8 & $13100 / 7900$ & $0.62:$ & $34500:$ & \\
Ne IV & 63.4 & $242.4 / 242.2$ & 1.11 & 3400 & $4000^{c}$ \\
\hline
\end{tabular}

${ }^{1}$ Values taken from Keenan et al. $(1996)^{a},(1997)^{b},(1998)^{c}$, $(1999)^{d}$.

: Large uncertainty.

* IP: Ionization potential.

the $N_{\mathrm{e}}$ given by Hyung \& Aller (1998) and Keenan et al. $(1996,1997,1998,1999)$ and therefore has been adopted to derive the $T_{\mathrm{e}}$ and abundances.

$N_{\mathrm{e}}$ derived from S III and Ar V differs by a large amount from the rest of the ions. This could be due to the fact that the argon line at $13.01 \mu \mathrm{m}$ and especially the sulfur line at $33.47 \mu \mathrm{m}$ are very noisy.

The $N_{\mathrm{e}}$ has been derived for several ions which have different ionization potential (IP). This value seems to be constant, about $4500 \mathrm{~cm}^{-3}$, there is no trend with IP.

\subsubsection{Electron temperature}

To derive $T_{\mathrm{e}}$ the ratio of lines originating from levels that differ by several electron volts are needed. An $N_{\mathrm{e}}=$ $4500 \mathrm{~cm}^{-3}$ has been assumed.

$T_{\mathrm{e}}$ has been derived for thirteen different ions and the values are shown in Table 6 . The temperatures are plotted versus the ionization potential in Fig. 2 . The curve shows a trend of $T_{\mathrm{e}}$ with IP.

The $T_{\mathrm{e}}$ derived from N II differs by $1400 \mathrm{~K}$ depending on whether the optical lines from Hyung \& Aller (1998) or Perinotto \& Corradi (1998) are used. Both values are shown in Fig. 2. The difference is mainly due to the flux difference of the $658.3 \mathrm{~nm}$ line, about $30 \%$. The observations correspond to different parts of the nebula. However, the $575.5 \mathrm{~nm}$ line fluxes are very similar, indicating that the 658.3 line discrepancy maybe asscribed to errors in flux determination. The ratio based on Perinotto \& Corradi's (1998) fluxes gives a $T_{\mathrm{e}}$ which better agrees with that of O II, that has a similar IP, and therefore was adopted to derive the curve in Fig. 2. The difference in derived $T_{\mathrm{e}}$ shows how differences in the fluxes can lead to noticeable differences in the reported temperature.

In Fig. 2 the values tabulated in Table 6 have been plotted with the exception of the NeIII $T_{\mathrm{e}}$. NeIII has been excluded because in other studies (Pottasch \& Beintema 1999; Bernard Salas et al. 2001) it usually gives 


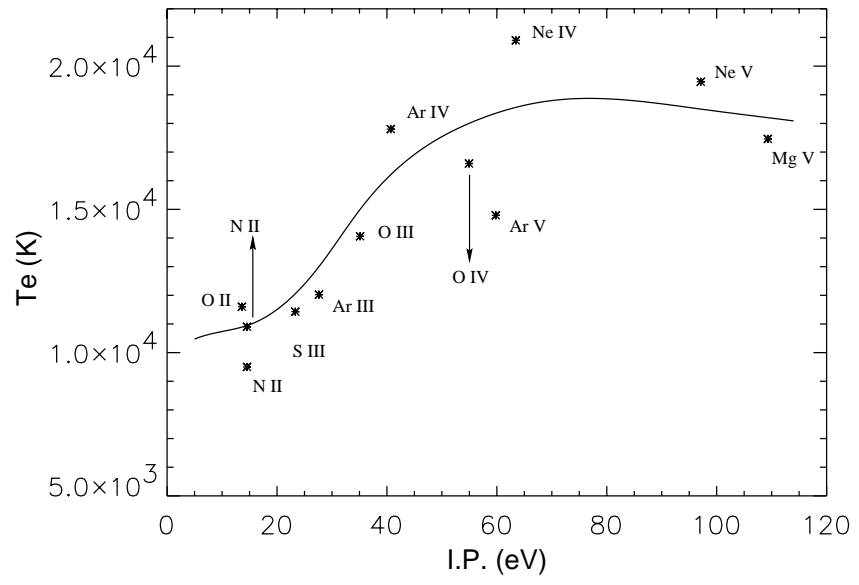

Fig. 2. Electron temperature versus ionization potential. The ratios used for each ion are indicated. The N II ratio plotted in this figure, has been derived using the line fluxes given by Perinotto \& Corradi (1998), see Table 6. The shape of the curve is in accordance with the expected physical behavior (see discussion Sect. 5.1.2).

Table 6. Electron temperature. $N_{\mathrm{e}}=4500 \mathrm{~cm}^{-3}$ is used.

\begin{tabular}{lccccc}
\hline \hline Ion & $\begin{array}{c}\text { IP } \\
(\mathrm{eV})\end{array}$ & $\begin{array}{c}\text { Lines used } \\
(\mathrm{nm})\end{array}$ & $\begin{array}{c}\text { Obs. } \\
\text { ratio }\end{array}$ & $\begin{array}{c}T_{\mathrm{e}} \\
(\mathrm{K})\end{array}$ & $\begin{array}{c}\text { Other } \\
\text { ref. }^{1}\end{array}$ \\
\hline O II & 13.6 & $732.0 / 372.6^{\star}$ & 0.177 & 11700 & $11700^{1 c}$ \\
N II & 14.5 & $575.5 / 658.4$ & 0.0143 & 9500 & \\
N II & 14.5 & $575.5 / 658.4$ & 0.0197 & $10900^{\dagger}$ & \\
S III & 23.3 & $631.2 / 18700$ & 0.088 & 11400 & \\
Ar III & 27.6 & $519.2 / 8990$ & 0.018 & 12000 & \\
O III & 35.1 & $436.3 / 500.7$ & 0.0169 & 14060 & \\
Ar IV & 40.7 & $726.3 / 471.1$ & 0.039 & 17800 & $17650^{1 a}$ \\
Ne III & 41.0 & $386.8 / 15500$ & 1.68 & 13800 & \\
O IV & 54.9 & $140.0 / 25900$ & 0.148 & 16600 & \\
Ar V & 59.8 & $700.5 / 7900$ & 0.39 & 14800 & \\
Ne IV & 63.4 & $472.4 / 242.2^{\star \star}$ & 0.012 & 20900 & $19800^{1 b}$ \\
Ne V & 97.1 & $342.6 / 24300$ & 0.95 & 19400 & \\
Mg V & 109.3 & $278.3 / 5610$ & $0.61:$ & 17500 & \\
\hline
\end{tabular}

${ }^{1}$ Values taken from Keenan et al. $(1997)^{a},(1998)^{b},(1999)^{c}$.

${ }^{\dagger}$ Using the line fluxes given by Perinotto \& Corradi (1998).

* The lines used are; $(732.0+773.0) /(372.6)$.

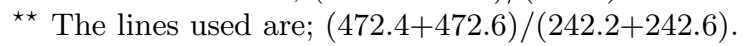

: Very noisy.

a lower $T_{\mathrm{e}}$. Probably this is due to the atomic parameter data. The $T_{\mathrm{e}}$ derived from the $\mathrm{S}$ III using the line ratio $631.2 \mathrm{~nm} / 33479 \mathrm{~nm}$ is not included because the errors are too large. Instead the ratio $631.2 / 18700$ has been used. It should also be noticed that the S III ratio is sensitive to the adopted $N_{\mathrm{e}}$ and this could explain the difference found in both $T_{\mathrm{e}}$. To derive chemical abundances $T_{\mathrm{e}}$ corresponding to the IP of each ion (given by the curve in Fig. 2) and $N_{\mathrm{e}}=4500 \mathrm{~cm}^{-3}$ have been assumed.

\subsection{Discussion}

The $N_{\mathrm{e}}$ sees to be constant and this could mean that the ejection of the outer layers is now at lower rates than it has been before, leading to an homogeneous stratification of the nebula.

A trend of $T_{\mathrm{e}}$ with IP is found. As previously discussed by Bernard Salas et al. (2001) for NGC 7027, this agrees with a simple picture of a nebula where the high ionization states are reached closer to the central star so they give higher temperatures, and the low states of ionization give lower $T_{\mathrm{e}}$ because they are formed further away. It is worth mention that the different values for the optical lines (of N II) given by Hyung \& Aller (1998) and Perinotto \& Corradi (1998) lead to different $T_{\mathrm{e}}$ which can slightly affect the relative abundances derived for low ionization potentials (IP) ions.

To derive the abundances for each ion a $T_{\mathrm{e}}$ has been assumed as determined by the curve in Fig. 2. The temperature assumption is more important for ions where the abundance has been derived using optical or ultraviolet data. Abundances derived from infrared lines are insensitive to the adopted temperature. As can be seen in Fig. 2 the trend at high IP is not as well determined as at low IP. For ions with low IP the abundance has been derived mainly using ultraviolet or optical data, that are sensitive to the $T_{\mathrm{e}}$ adopted, but where the curve is well determined. On the other hand abundances of ions at high IP have been derived from infrared lines and therefore are insensitive to the adopted $T_{\mathrm{e}}$.

\section{Chemical abundances}

To derive the ionic abundances a $N_{\mathrm{e}}=4500 \mathrm{~cm}^{-3}$ (see Sect. 5.1.1) an a $T_{\mathrm{e}}$ according to the IP of each ion (see Sect. 5.1.2) have been assumed.

\subsection{Analysis}

The element abundances for carbon, nitrogen, oxygen, neon, magnesium, argon and sulfur have been derived. Unfortunately for silicon and chlorine only one stage of ionization was measured which is not enough to properly derive the abundance. The determination of the ionization correction factor (ICF) is too uncertain. The ions for which abundances have been derived are listed in Table 7.

The equation used to determine these ionic abundances is:

$\frac{N_{\text {ion }}}{N_{\mathrm{p}}}=\frac{I_{\text {ion }}}{I_{\mathrm{H}_{\beta}}} N_{\mathrm{e}} \frac{\lambda_{\mathrm{ul}}}{\lambda_{\mathrm{H}_{\beta}}} \frac{\alpha_{\mathrm{H}_{\beta}}}{A_{\mathrm{ul}}}\left(\frac{N_{\mathrm{u}}}{N_{\text {ion }}}\right)^{-1}$.

In the equation $N_{\mathrm{p}}$ represents the density of ionized hydrogen; $I_{\mathrm{ion}} / I_{\mathrm{H}_{\beta}}$ is the measured intensity of the ionic line, normalized to $\mathrm{H}_{\beta} ; \lambda_{\mathrm{ul}}$ is the wavelength of the line and $\lambda_{\mathrm{H}_{\beta}}$ is the wavelength of $\mathrm{H}_{\beta} ; \alpha_{\mathrm{H}_{\beta}}$ is the effective recombination coefficient for $\mathrm{H}_{\beta} ; A_{\mathrm{ul}}$ is the Einstein spontaneous transition rate for the line and finally $N_{\mathrm{u}} / N_{\text {ion }}$ is the ratio 
Table 7. Ionic and element abundances.

\begin{tabular}{|c|c|c|c|c|c|}
\hline Ion & $\lambda(\mathrm{nm})$ & $\Omega^{\star}$ & $N_{\text {ion }} / N_{\mathrm{p}}$ & $\mathrm{ICF}^{1}$ & $N_{\text {el. }} / N_{\mathrm{p}}$ \\
\hline $\mathrm{He}^{+}$ & 587.7 & & $0.0554^{\sharp}$ & & \\
\hline $\mathrm{He}^{++}$ & 468.6 & & $0.0639^{\sharp}$ & 1 & 0.119 \\
\hline $\mathrm{C}^{+}$ & 232.7 & $\mathrm{a}$ & $1.06(-4)$ & & \\
\hline $\mathrm{C}^{++}$ & 190.9 & $\mathrm{~b}$ & $5.68(-4)$ & & \\
\hline $\mathrm{C}^{3+}$ & 154.9 & $\mathrm{~b}$ & $3.75(-5)$ & 1.01 & $7.19(-4)$ \\
\hline $\mathrm{N}^{0}$ & $519.8,520.0$ & $\mathrm{~b}$ & $7.88(-6)$ & & \\
\hline $\mathrm{N}^{+}$ & 658.3 & $\mathrm{c}$ & $1.14(-5)$ & & \\
\hline $\mathrm{N}^{+}$ & $658.3^{\dagger}$ & $\mathrm{c}$ & $7.80(-6)$ & & \\
\hline $\mathrm{N}^{+}$ & 575.5 & $\mathrm{c}$ & $8.21(-5)$ & & \\
\hline $\mathrm{N}^{++}$ & 175.0 & $\mathrm{a}$ & $3.09(-4)$ & & \\
\hline $\mathrm{N}^{3+}$ & 148.5 & $\mathrm{~b}$ & $9.39(-5)$ & & \\
\hline $\mathrm{N}^{4+}$ & 123.9 & $\mathrm{~b}$ & $1.50(-5)$ & 1 & $4.37(-4)$ \\
\hline $\mathrm{O}^{0}$ & 630.0 & $\mathrm{~b}$ & $2.01(-5)$ & & \\
\hline $\mathrm{O}^{+}$ & $372.7,373.0$ & d & $3.54(-5)$ & & \\
\hline $\mathrm{O}^{++}$ & $500.8,436.3$ & c & $1.82(-4)$ & & \\
\hline $\mathrm{O}^{3+}$ & 25900 & $\mathrm{e}$ & $1.06(-4)$ & 1.1 & $3.78(-4)$ \\
\hline $\mathrm{Ne}^{+}$ & 12810 & $\mathrm{f}$ & $1.10(-5)$ & & \\
\hline $\mathrm{Ne}^{++}$ & 15500 & $\mathrm{~g}$ & $4.10(-5)$ & & \\
\hline $\mathrm{Ne}^{3+}$ & $242.5,472.5$ & h,b & $2.26(-5)$ & & \\
\hline $\mathrm{Ne}^{4+}$ & 14320 & $\mathrm{c}$ & $8.99(-6)$ & & \\
\hline $\mathrm{Ne}^{4+}$ & 24300 & $\mathrm{c}$ & $1.65(-5)$ & & \\
\hline $\mathrm{Ne}^{5+}$ & 7650 & $\mathrm{e}$ & $1.02(-5)$ & 1 & $1.10(-4)$ \\
\hline $\mathrm{Mg}^{+}$ & 280.0 & $\mathrm{i}$ & $7.56(-9)$ & & \\
\hline $\mathrm{Mg}^{4+}$ & 5610 & $\mathrm{j}$ & $3.42(-6)$ & - & - \\
\hline $\mathrm{S}^{+}$ & $671.6,673.1$ & $\mathrm{k}$ & $3.24(-7)$ & & \\
\hline $\mathrm{S}^{++}$ & 18710 & 1 & $1.80(-6)$ & & \\
\hline $\mathrm{S}^{3+}$ & 10510 & $\mathrm{~m}$ & $1.10(-6)$ & 1.47 & $4.72(-6)$ \\
\hline $\mathrm{Cl}^{++}$ & $553.8,551.8$ & $\mathrm{~b}$ & $4.51(-8)$ & & \\
\hline $\mathrm{Ar}^{+}$ & 6980 & $\mathrm{n}$ & $3.58(-7)$ & & \\
\hline $\mathrm{Ar}^{++}$ & 8990 & 1 & $1.31(-6)$ & & \\
\hline $\mathrm{Ar}^{3+}$ & $471.1,474.0$ & o & $7.09(-7)$ & & \\
\hline $\mathrm{Ar}^{4+}$ & $7900,700.6$ & 1 & $2.38(-7)$ & & \\
\hline $\mathrm{Ar}^{5+}$ & 4527 & $\mathrm{p}$ & $3.58(-7)$ & 1.07 & $3.17(-6)$ \\
\hline
\end{tabular}

${ }^{1} \mathrm{ICF}=$ Ionization Correction Factor.

$\sharp$ Using the predictions for the helium emission lines given by Benjamin et al. (1999).

$\dagger$ Value from Perinotto \& Corradi (1998).

${ }^{\star}$ References for the collisional strengths $(\Omega)$ : a) Blum \& Pradhan (1992), b) Mendoza (1983), c) Lennon \& Burke (1994), d) McLaughlin \& Bell (1993), e) Zhang et al. (1994), f) Saraph \& Tully (1994), g) McLaughlin \& Bell (2000), h) Ramsbottom et al. (1998), i) Sigut \& Pradhan (1995), j) Butler \& Zeippen (1994), k) Keenan et al. (1996), l) Galavís et al. (1995), m) Saraph \& Storey (1999), n) Pelan \& Berrington (1995), o) Ramsbottom et al. (1997), p) Saraph \& Storey (1996).

of the population of the level from which the line originates to the total population of the ion. The collisional strengths $(\Omega)$ used to derive the population of the levels are tabulated in Col. 3 of Table 7. Collisional strengths derived from the Iron Project were preferred, when not available, the most recent ones were chosen from the literature. The transition probabilities used are those from Mendoza (1983) for all ions except for $\mathrm{Ne} V$ where the ones by Galavís et al. (1997) were used.
As can be seen in Table 7 the ICF is 1 or 1.1 in all cases but sulfur. This shows how powerful it can be to combine infrared, optical and ultraviolet data in order to get reliable abundances. The ICF for sulfur is bigger because some contribution from $\mathrm{S}^{4+}$ and $\mathrm{S}^{5+}$ is expected. The ICF were calculated on a purely empirical basis using the known distribution of neon ions (with varying IP) as an example.

\subsection{Discussion of the abundances}

The abundances derived have been compared to those found in the Sun, O, B stars and previous literature (see Table 8 for references).

The helium and carbon abundances agree with those found by Kwitter \& Henry (1996). The carbon abundance found by Hyung \& Aller (1998) seems too small. The nitrogen is half that found by the same authors. The ICF used by Hyung \& Aller (1998) seems too large. On the other hand the oxygen and neon abundances agree quite well. The sulfur abundance is higher than the ICFMethod and Model employed by Hyung \& Aller (1998), but four and three times smaller than that of the Sun and O, B stars. For this element a high ICF of 1.47 (see Table 7) was needed because contribution from $\mathrm{S}^{4+}$ and $\mathrm{S}^{5+}$ is expected. The IP of these ions $(47.3$ and $72.7 \mathrm{eV}$ respectively) is still in the limit where the radiation of the central star can ionize them. The reason for the lower abundance of sulfur is not clear. It can not be due to the ICF used because to match the solar sulfur abundance an ICF of 5.8 (or ICF $=2.6$ to match the $\mathrm{O}, \mathrm{B}$ stars) should be used which is definitively too high. Finally the argon abundance from Hyung \& Aller (1998) is smaller than the present study. The abundances in Perinotto \& Corradi (1998) constitute an average of various regions of the nebula. Since they have been weighted to the local surface brightness, they refer to the brightest positions which correspond to the center, $p 1$ and $n 1$ (Sect. 4.3 or see Perinotto \& Corradi (1998) for details) and are therefore comparable to ISO and IUE observations. The helium, oxygen, argon abundances agree with those of this paper. The neon and sulfur abundances are lower than the present study, but agree better than those of the models. The nitrogen abundance from Perinotto \& Corradi (1998) is much higher but they only measured N II, and used a highly uncertain ICF. From the C, N, O elements, only oxygen is less abundant than in the Sun and O, B stars. Maybe some oxygen has been converted into carbon and nitrogen via the CNO cycle. Neon and argon are similar to O, B stars which could mean that the nebula hasn't formed any of these elements during its evolution. It is interesting to compare the ratio of carbon, oxygen and/or nitrogen. This is shown in Table 9.

The N/O ratio is 3 times bigger than in NGC 7027 and 9 times bigger than in the Sun and O, B stars. A less pronounced difference occurs for the $\mathrm{C} / \mathrm{O}$ ratio. Probably the oxygen has been converted into nitrogen and to some 
Table 8. Comparison of abundances in NGC 2440.

\begin{tabular}{|c|c|c|c|c|c|c|c|}
\hline \multirow[t]{2}{*}{ Element } & \multirow{2}{*}{$\begin{array}{l}\text { Present } \\
\text { abund. }\end{array}$} & \multirow[t]{2}{*}{$\operatorname{Sun}^{1}$} & \multirow[t]{2}{*}{ O, B Stars ${ }^{2}$} & \multicolumn{2}{|c|}{ Hyung \& Aller ${ }^{3}$} & \multirow{2}{*}{$\begin{array}{l}\text { Kwitter } \\
\text { Model }\end{array}$} & \multirow{2}{*}{$\begin{array}{l}\text { Perinotto } 5 \\
\& \text { Corradi }\end{array}$} \\
\hline & & & & ICF-M & Model & & \\
\hline Helium & 0.119 & 0.098 & & 0.126 & 0.11 & 0.12 & 0.124 \\
\hline Carbon & $7.2(-4)$ & $3.5(-4)$ & $1.7(-4)$ & $3.4(-4)$ & $4.0(-4)$ & $6.3(-4)$ & \\
\hline Nitrogen & $4.4(-4)$ & $9.3(-5)$ & $6.5(-5)$ & $1.0(-3)$ & $9.0(-4)$ & $8.2(-4)$ & $7.0(-4)$ \\
\hline Oxygen & $3.8(-4)$ & $7.4(-4)$ & $4.2(-4)$ & $4.4(-4)$ & $3.5(-4)$ & $5.7(-4)$ & $3.8(-4)$ \\
\hline Neon & $1.1(-4)$ & $1.2(-4)$ & $1.2(-4)$ & $9.5(-5)$ & $7.0(-5)$ & $7.4(-5)$ & $6.0(-5)$ \\
\hline Magnesium & - & $3.8(-5)$ & $2.4(-5)$ & $2.0(-6)$ & $2.0(-6)$ & & \\
\hline Silicon & - & $3.5(-5)$ & $2.4(-5)$ & $1.7(-6)$ & $1.9(-6)$ & & \\
\hline Sulfur & $4.7(-6)$ & $1.9(-5)$ & $1.2(-5)$ & $1.7(-6)$ & $2.0(-6)$ & & $3.1(-6)$ \\
\hline Chlorine & - & $1.9(-7)$ & $1.9(-7)$ & $1.3(-7)$ & $1.2(-7)$ & & \\
\hline Argon & $3.2(-6)$ & $3.6(-6)$ & & $2.0(-6)$ & $2.2(-6)$ & & $2.6(-6)$ \\
\hline
\end{tabular}

${ }^{1}$ Solar abundance from Grevesse et al. (1993) and Anders \& Grevesse (1989).

2 O, B star abundances are the average of Gies \& Lambert (1992) and Killian-Montenbruck et al. (1994).

3 Abundances by Hyung \& Aller (1998), determined using the ICF-Method and modeling.

${ }^{4}$ From Kwitter \& Henry (1996).

${ }^{5}$ From Perinotto \& Corradi (1998).

Table 9. Comparison of the abundance ratios of $\mathrm{C}, \mathrm{N}$ and $\mathrm{O}$.

\begin{tabular}{ccccc}
\hline \hline & NGC 2440 & NGC 7027 & Sun & O Stars \\
\hline $\mathrm{N} / \mathrm{O}$ & 1.2 & 0.39 & 0.15 & 0.15 \\
$\mathrm{C} / \mathrm{O}$ & 1.9 & 1.5 & 0.48 & 0.42 \\
$\frac{(\mathrm{C}+\mathrm{N}+\mathrm{O})}{\mathrm{H}}$ & $1.5(-3)$ & $1.2(-3)$ & $1.2(-3)$ & $6.6(-4)$ \\
\hline
\end{tabular}

${ }^{1}$ From Bernard Salas et al. (2001).

extent into carbon as well. The $\mathrm{C}+\mathrm{N}+\mathrm{O} / \mathrm{H}$ ratio is twice as big as in any other source. The progenitor star of NGC 2440 must be much more massive than the Sun or NGC 7027 (3-4 $M_{\odot}$, Bernard Salas et al. 2001). This is also supported by the helium abundance which is also larger than any of these comparison sources.

\section{Conclusions}

The combined use of ISO, IUE and optical data is a powerful tool to derive NGC 2440 's elemental abundances.

The density seems to be constant (see Table 5) across the nebula because line ratios of ions with different IP that are probably formed in different regions give the same electron density. The nebula's size is expected to increase with time as more layers are ejected. In order to keep a constant density in an expanding nebula, the ejection should occur at continously lower rates. The electron temperature increases with ionization potential (see Fig. 2). This has previously been detected for NGC 7027 (Bernard Salas et al. 2001), NGC 6302 (Pottasch \& Beintema 1999) and NGC 6537 (Pottasch et al. 2000). A simple picture of a planetary nebula is that close to the central star there exist more high energy UV photons than in the outer regions. Thus high IP ions are formed close to the central star only and in an enviroment of high temperature. Low stages of ionization require lower UV photons and conse- quently give lower temperature. From Table 9 it is clear that the CNO cycle has converted much of the oxygen into nitrogen and to some extent into carbon. The $\mathrm{C}+\mathrm{N}+\mathrm{O} / \mathrm{H}$ ratio is larger than the Sun's and NGC 7027. A study by Bernard Salas et al. (2001) of NGC 7027 reveals a progenitor's mass of about 3-4 $M_{\odot}$. Therefore NGC 2440 's progenitor must have been more massive. This is supported by the fact that the carbon, nitrogen and oxygen abundances (Table 8) are, although lower, close to those of more massive $\mathrm{O}, \mathrm{B}$ stars.

Acknowledgements. We are grateful to the referee, Prof. M. Perinotto whose comments have result in an improvement of the paper. IA3 is a joint development of the SWS consortium. Contributing institutes are SRON, MPE, KUL and the ESA Astrophysics Division.

\section{References}

Anders, E., \& Grevesse, N. 1989, Geochem. Cosmo., 53, 197 Benjamin, R. A., Skillman, E. D., \& Smits, D. P. 1999, ApJ, 514,307

Bernard Salas, J., Pottasch, S. R., Beintema, D. A., \& Wesselius, P. R. 2001, A\&A, 367, 949

Blum, R. D., \& Pradhan, A. K. 1992, ApJS, 80, 452

Butler, K., \& Zeippen, C. J. 1994, A\&AS, 108, 1

Fluks, M. A., Plez, B., Thé, P. S., et al. 1994, A\&AS, 105, 311

Galavís, M. E., Mendoza, C., \& Zeippen, C. J. 1995, A\&AS, 111,347

Galavís, M. E., Mendoza, C., \& Zeippen, C. J. 1997, A\&AS, 123,159

de Graauw, T., Haser, L. N., Beintema, D. A., et al. 1996, A\&A, 315, L49

Gies, D. R., \& Lambert D. L. 1992, ApJ, 387, 673

Grevesse, N., \& Noels, A. 1993, in Origin of the Elements, ed. N. Prantos, et al. (Cambridge University Press), 15

Hummer, D. G., \& Storey, P. J. 1987, MNRAS, 224, 801

Hyung, S., \& Aller, L. H. 1998, PASP, 110, 466

Keenan, F. P., Aller, L. H., Bell, K. L., et al. 1996, MNRAS, 281, 1073 
Keenan, F. P., McKenna, F. C., Bell, K. L., et al. 1997, ApJ, 487, 457

Keenan, F. P., Aller, L. H., Bell, K. L., et al. 1998, MNRAS, 295,683

Keenan, F. P., Aller, L. H., Bell, K. L., et al. 1999, MNRAS, 304, 27

Kessler, M. F., Steinz, J. A., Anderegg, M. E., et al. 1996, A\&A, 315, L27

Killian-Montenbruck, J., Grehen, T., \& Nissen, P. E. 1994, ApJ, 291, 757

Kwitter, K. B., \& Henry, R. B. C. 1996, ApJ, 473, 304

Lennon, D. J., \& Burke, V. M. 1994, A\&AS, 103, 273

McLaughlin, B. M., \& Bell, K. L. 1993, ApJ, 408, 753

McLaughlin, B. M., \& Bell, K. L. 2000, J. Phys. B: At. Mol. Opt. Phys., 33, 597

Mendoza, C. 1983, in IAU Symp. 103, Planetary Nebulae, ed. D. R. Flower, 143

Peimbert 1978, IAU Symp. 76, Plan. Neb., ed. Y. Terzian, 215

Pelan, J., \& Berrington, K. A. 1995, A\&AS, 110, 209

Perinotto, M., \& Corradi, R. L. M. 1998, A\&A, 332, 721

Pottasch, S. R. 1984, Planetary Nebulae, D-Reidel, vol. 107
Pottasch, S. R., Preite-Martinez, A., Olnon, F. M., Mo, J. E., \& Kingma, S. 1986, A\&A, 161, 363

Pottasch, S. R., \& Beintema, D. A. 1999, A\&A, 347, 975

Pottasch, S. R., Beintema, D. A., \& Feibelman, W. A. 2000, A\&A, 363, 767

Ramsbottom, C. A., Bell, K. L., \& Keenan, F. P. 1997, MNRAS, 284, 754

Ramsbottom, C. A., Bell, K. L., \& Keenan, F. P. 1998, MNRAS, 293, 233

Rowland, N., Houck, J. R., Skrutskie, M. F., \& Shure, M. 1993, PASP, 105, 1287

Saraph, H. E., \& Tully, J. A. 1994, A\&AS, 107, 29

Saraph, H. E., \& Storey, P. J. 1996, A\&AS, 115, 151

Saraph, H. E., \& Storey, P. J. 1999, A\&AS, 134, 369

Sigut, T. A. A., \& Pradhan, A. K. 1999, J. Phys. B: At. Mol. Opt. Phys., 28, 4879

Shields, G. A., Aller, L. H., Keyes, C. D., \& Czyzak, S. J. 1981, ApJ, 248, 569

Walker, M. F., \& Aller, L. H. 1970, ApJ, 151, 917

Zhang, H. L., Graziani, M., \& Pradhan, A. K. 1994, A\&A, 283, 319 